\title{
Metabolic syndrome in psoriasis
}

\author{
Anna Szponar-Bojda, Dorota Krasowska, Aldona Pietrzak, Grażyna Chodorowska
}

Department of Dermatology, Venereology and Pediatric Dermatology, Medical University of Lublin, Poland Head: Prof. Grażyna Chodorowska MD, PhD

Postep Derm Alergol 2012; XXIX, 5: 356-362

DOI: 10.5114/pdia.2012.31488

\begin{abstract}
Introduction: Recent years' studies have revealed a link between the metabolic syndrome and psoriasis. The common elements of pathogenesis of both diseases, primarily associated with severe inflammation, may be responsible for an increased incidence of metabolic syndrome components in psoriasis patients.

Aim: To evaluate the prevalence of selected risk factors for the metabolic syndrome in patients with psoriasis and possible involvement of these factors in the severity of psoriasis.

Material and methods: The study included 117 adult psoriatic patients compared with a control group. The severity of psoriasis was evaluated using the PASI and mNAPSI score. Basic laboratory tests were performed. The body mass index (BMI), De Ritis ratio and blood pressure were determined for each patient.

Results: In the psoriasis group, excess body weight and abnormal values of BMI were significantly more frequent. Body mass index and body weight were significantly higher in patients with the calculated De Ritis ratio than in other subjects. The levels of erythrocyte sedimentation rate and C-reactive protein were significantly higher in the psoriasis group and positively correlated with BMI. Compared with controls, patients with psoriasis had significantly higher values of systolic and diastolic blood pressure, with no correlation with the severity of psoriasis evaluated using the PASI and mNAPSI score.

Conclusions: The results obtained in our study indicate that psoriatic patients appear to be at a higher risk of the metabolic disorders such as hypertension, obesity, liver dysfunction, increased inflammatory parameters than healthy subjects, irrespective of the full manifestation of the metabolic syndrome. We suggest that patients with psoriasis should be routinely screened for the presence of the above-mentioned disorders.
\end{abstract}

Key words: psoriasis, metabolic syndrome, De Ritis ratio, C-reactive protein, hypertension.

\section{Introduction}

In 1988, the term "syndrome X" was introduced, stressing that several metabolic disorders commonly cluster together, including hyperinsulinemia, insulin resistance, dyslipidemia, hypertension, hyperglycemia and coronary heart disease [1]. Since then, the definition of the syndrome has undergone several modifications and the criteria for the diagnosis have changed. The clustering of metabolic risk factors is now known as the metabolic syndrome. In 2004, the definition currently used was established by the International Diabetes Federation [2]. The abdominal obesity, i.e. waist circumference $\geq 80 \mathrm{~cm}$ for European women and $\geq 94 \mathrm{~cm}$ for European men, is the main diagnostic criterion for metabolic syndrome. In the earlier definitions, the body mass index (BMI) was used as an indicator of obesity and race was not taken into account while realigning the results [2]. According to the recent guidelines, visceral obesity can be diagnosed based on $\mathrm{BMI} \geq 30 \mathrm{~kg} / \mathrm{m}^{2}$ without abdominal circumference measurements. Moreover, it is necessary to meet two of four following criteria to diagnose the metabolic syndrome:

- raised levels of triglycerides $\geq 1.7 \mathrm{mmol} / \mathrm{l}(150 \mathrm{mg} / \mathrm{dl}$ ) or specific treatment for this lipid abnormality,

- reduced levels of $\mathrm{HDL}$ cholesterol $<1.03 \mathrm{mmol} / \mathrm{l}$ (40 mg/dl) in men and < $1.29 \mathrm{mmol} / \mathrm{l}(50 \mathrm{mg} / \mathrm{dl})$ in women or specific treatment for this lipid abnormality,

- raised systolic blood pressure $\geq 130 \mathrm{~mm}$ Hg or diastolic blood pressure $\geq 85 \mathrm{~mm} \mathrm{Hg}$ or treatment of previously diagnosed hypertension,

- raised fasting plasma glucose $\geq 5.6 \mathrm{mmol} / \mathrm{dl}(100 \mathrm{mg} / \mathrm{dl})$ or previously diagnosed type 2 diabetes [3].

Recent years' studies have revealed a link between metabolic syndrome and psoriasis [4-6]. The common ele-

Address for correspondence: Anna Szponar-Bojda MD, PhD, Department of Dermatology, Venereology and Pediatric Dermatology, Medical University of Lublin, 13 Radziwillowska St, 20-080 Lublin, Poland, phone: +48 8153236 47, e-mail: anna.szponar@wp.pl 
ments of the pathogenesis of both diseases, primarily associated with severe inflammation, may be responsible for an increased incidence of metabolic syndrome components in patients with psoriasis. Many studies suggest that proinflammatory cytokines may affect the development of atherosclerotic lesions in blood vessels and insulin resistance $[4,7]$. Psoriasis is a chronic inflammatory skin disease characterized by immunological abnormalities and increased release of inflammatory mediators, including tumour necrosis factor $\alpha$ (TNF- $\alpha)$, interleukin-1 (IL-1), interleukin-8 (IL-8) [8], which simultaneously stimulates the development of metabolic disorders, e.g. obesity. It is well known that the adipose tissue acts as an active endocrine organ, secreting adipocytokines, including interleukin-6 (IL-6) and TNF- $\alpha$ [9]. Adipocytes release proinflammatory cytokines that can exacerbate the ongoing inflammation, worsen psoriasis and increase the risk of metabolic syndrome. The most common disorders whose pathogenesis may have a relationship with psoriasis are dyslipidemia, carbohydrate metabolism disorders, atherosclerosis, hypertension, abdominal obesity, diabetes and consequently the metabolic syndrome and cardiovascular system diseases [10-12].

\section{Material and methods}

The study involved 117 adult patients aged 18 to 60 years, including 25 women and 92 men with psoriasis (PASI range 1.2-38.4). They were compared with a group of 40 age- and weight-matched healthy controls with no family history of psoriasis. Patients with pustular psoriasis, a history of connective tissue diseases, viral hepatitis and cancer as well as smokers and alcohol abusers were excluded from the study. The severity of psoriatic skin lesions was evaluated using the Psoriasis Area and Severity Index (PASI) and modified Nail Psoriasis Severity Index (mNAPSI). Laboratory tests such as blood cell morphology, blood glucose level, alanine aminotransferase (AIAT) and aspartate aminotransferase (AspAT) levels, total bilirubin level, erythrocyte sedimentation rate (ESR), C-reactive protein (CRP) were performed. The height, weight, BMI, De Ritis ratio and blood pressure were determined for each patient. In the group of patients with psoriasis, 11 people had psoriatic arthritis, 70 people manifested abnormal blood pressure or were treated for hypertension. The research protocol was approved by the Local Bioethical Committee of the Medical University of Lublin (KE-0254/136/2009). Each patient was informed about the aim of the study and signed the informed consent. The classification of psoriasis was performed according to the guidelines of the International Psoriasis Council of 2005 [13].

\section{Statistical analysis}

A computer program Statistica 8.0 PL was used for statistical analysis. The arithmetic mean (AM) and standard deviation (SD) were used in the structural analysis and minimum and maximum values were calculated. The results were presented with the use of basic parameters of descriptive statistics, such as the median (M) and the value of the lower and upper quartile. The distribution of variables was checked with the Shapiro-Wilk test. Since the analysed variables were not normally distributed, nonparametric tests were used. Intergroup differences were compared using the Mann-Whitney $U$ test whereas the correlation between variables was assessed with the Spearman's rank correlation coefficient. A significance level of $p \leq 0.05$ was accepted as statistically significant.

\section{Results}

The demographic data of patients and controls are presented in Table 1 . The study included 117 adult psoriatic patients aged from 18 to 60 years, mean $46.16 \pm 13.48$. The largest group consisted of patients aged from 40 to 60 years (68.37\%, 80 subjects). The subjects in the contro group were from 21 to 59 years old, mean $44.25 \pm 15$.2. The ages of in both groups were comparable. Analysis of the PASI score and mNAPSI values are presented in Table 2. In the group of psoriatic patients, BMI ranged between 18.12 and $42.19 \mathrm{~kg} / \mathrm{m}^{2}$; mean $26.89 \pm 4.89 \mathrm{~kg} / \mathrm{m}^{2}$. In the control group, mean BMI was lower: $25.69 \pm 3.72 \mathrm{~kg} / \mathrm{m}^{2}$, but the difference was not statistically significant $(p=0.29)$.

In patients with psoriasis, excess body weight was observed in 72 patients (61.5\%); 44 patients (37.6\%) were overweight (BMI $25-29.9 \mathrm{~kg} / \mathrm{m}^{2}$ ) and 28 (23.9\%) were obese $\left(\mathrm{BMI} \leq 30 \mathrm{~kg} / \mathrm{m}^{2}\right)$. Among patients with obesity, 20 had class I obesity (BMI 30-34.9 kg/m²), 4 - class II obesity (BMI $35-39.9 \mathrm{~kg} / \mathrm{m}^{2}$ ), and 4 patients class III obesity $\left(\mathrm{BMI} \geq 40 \mathrm{~kg} / \mathrm{m}^{2}\right)$. The structure of the psoriasis group by $\mathrm{BMI}$ is presented in Figure 1. In the control group, overweight (BMI $25-29.9 \mathrm{~kg} / \mathrm{m}^{2}$ ) was diagnosed in 14 patients (35\%) and obesity $\left(\mathrm{BMI} \leq 30 \mathrm{~kg} / \mathrm{m}^{2}\right)$ in 6 patients $(15 \%)$ Abnormal values of BMI were significantly more frequent

Table 1. Demographic structure of the studied group and control group

\begin{tabular}{lcccc}
\hline \multirow{2}{*}{ Sex } & \multicolumn{2}{c}{ Patients with psoriasis } & \multicolumn{2}{c}{ Control group } \\
\cline { 2 - 5 } & $n$ & $\%$ & $n$ & $\%$ \\
\hline Women & 25 & $21.4 \%$ & 11 & $27.5 \%$ \\
\hline Men & 92 & $78.6 \%$ & 29 & $72.5 \%$ \\
\hline Total & 117 & $100 \%$ & 40 & $100 \%$ \\
\hline
\end{tabular}

Table 2. The severity of psoriasis by PASI and mNAPSI score

\begin{tabular}{lcccc}
\hline Indicator & Min & Max & AM & SD \\
\hline PASI & 1.2 & 38.4 & 13.92 & 7.26 \\
\hline mNAPSI & 0 & 20 & 3.85 & 4.3 \\
\hline
\end{tabular}




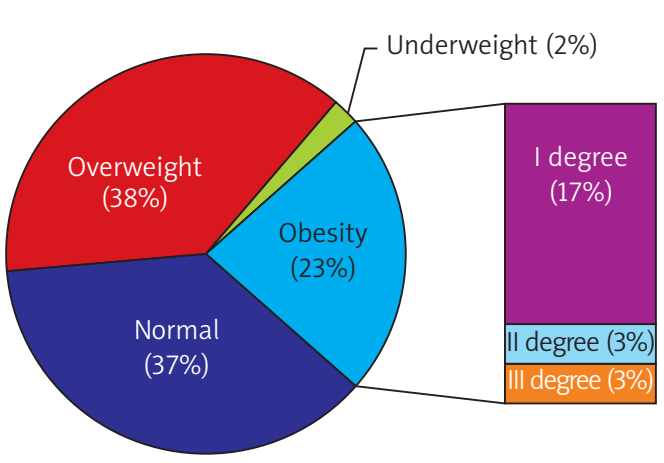

Figure 1. Body mass index in patients with psoriasis

in the group with psoriasis - $72(61.5 \%)$ vs. $20(50 \%)$ (odds ratio $(\mathrm{OR})=1.6)$.

In the group with psoriasis, 23 subjects (19.66\%) were treated for hypertension, and 47 patients (40.17\%) manifested abnormal blood pressure (systolic blood pressure $\geq 130 \mathrm{~mm} \mathrm{Hg}$, diastolic blood pressure $\geq 85 \mathrm{~mm} \mathrm{Hg}$ ). Compared with controls, patients with psoriasis had significantly higher values of systolic and diastolic blood pressure $(p=0.0004, Z=3.47$ and $p=0.036, Z=2.09)$.

The structure of the psoriasis group by co-morbidities is presented in Figure 2. Some psoriatic patients showed elevated blood glucose levels and glucose intolerance. However, compared with the control group, the difference was not statistically significant ( $p=0.86$ ).

Parameters of inflammation were significantly elevated in more than half of the psoriatic patients. The serum levels of ESR and CRP were significantly higher in the group with psoriasis than in the control group ( $p=0.0007$, $Z=3.3$ and $p=0.0004, Z=3.4$ ). The positive correlation between the parameters of inflammation (ESR: $r=0.02$, $R=0.20$, CRP: $p=0.005, R=0.25$ ) and BMI was observed in patients with psoriasis and in the control group.

In most patients, the levels of liver enzymes were normal or mildly elevated. We analyzed the De Ritis ratio as a parameter with high diagnostic usefulness in patients with slightly elevated transaminase levels. In the group with psoriasis, 21 subjects (17.95\%) manifested elevated AIAT and/or AspAT levels and the De Ritis ratio was calculated in these patients. Body mass index and body weight were significantly higher in patients with the calculated De Ritis ratio than in other subjects ( $p=0.03$, $Z=2.08$ and $p=0.01, Z=2.35$, respectively).

The relationship between psoriasis severity and selected clinical features was analyzed. The correlation between the PASI score and other parameters, such as BMI $(p=0.61)$, ESR levels $(p=0.38, R=0.08), \operatorname{CRP}(p=0.12$, $R=0.14)$, AlAT $(p=0.59, R=-0.04)$ and AspAT $(p=0.28$, $R=0.1)$ or total bilirubin $(p=0.45, R=-0.07)$ was not observed.

No significant differences in the PASI score were observed between the patients with psoriasis and coexisting hypertension versus those with psoriasis and nor-

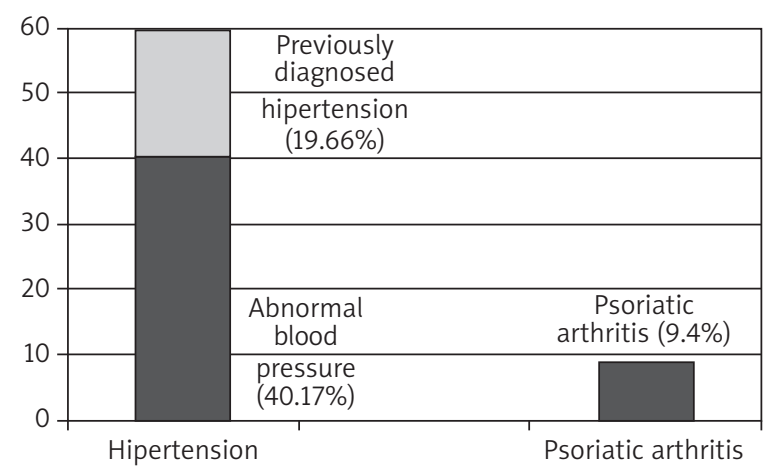

Figure 2. Comorbidities in patients with psoriasis

mal blood pressure $(p=0.31)$. Moreover, no significant correlation was found between the PASI score and systolic and diastolic blood pressure values $(R=0.03, p=0.7$ and $R=0.09, p=0.29)$. There were no statistically significant differences in nail psoriasis manifestation (mNAPSI) between patients with hypertension and patients with normal blood pressure $(p=0.65)$. Likewise, no significant relationship between mNAPSI values and systolic and diastolic pressure was demonstrated ( $R=0.02, p=0.82$ and $R=0.11, p=0.21$, respectively).

There was no difference in psoriasis severity (PASI) between patients with psoriasis and those with concomitant psoriasis vulgaris and psoriatic arthritis $(p=0.27)$, whereas patients with concomitant psoriatic arthritis manifested significantly higher mNAPSI values than subjects without psoriatic arthritis $(p=0.016$, $Z=-2.4$ ). Moreover, the higher the PASI score, the higher the mNAPSI score was observed $(p=0.002, R=0.27)$.

\section{Discussion}

In recent years, increased incidences of metabolic disorders have been observed in patients with psoriasis, including elevated levels of glucose, insulin resistance, hypertension, abdominal obesity or dyslipidemia [14], which constitute the metabolic syndrome, or those resulting from other diseases, e.g. cardiovascular diseases [15], type 2 diabetes, Crohn's disease [4]. The literature data indicate an increasing interest in liver dysfunction and the development of non-alcoholic fatty liver disease (NAFLD) in patients with psoriasis. The relationship between the prevalence of psoriasis and related co-morbidities is likely to be associated with chronic inflammation, underlying these diseases [16].

The pathogenesis of the metabolic syndrome has not been completely explained, but the abdominal obesity and associated insulin resistance are considered the most important factors. In the course of disease, the endothelium damage, increased platelet aggregation and excessive growth factors develop. The most common disorder of metabolic syndrome is hypertension, strictly associated with hyperinsulinemia and sympathetic nervous sys- 
tem activation. Moreover, severe inflammation appears to be relevant considering the demonstrated link between the metabolic syndrome risk factors (e.g. hypertension, dyslipidemia, hyperglycemia) and inflammatory parameters, such as CRP [1]. It is believed that, if left untreated, the metabolic syndrome can lead to the development of type 2 diabetes, severe atherosclerosis and cardiovascular diseases. It is estimated that metabolic syndrome occurs in approximately $20 \%$ of the Polish population [17].

Based on numerous reports it can be concluded that abnormal body weight and high BMI are much more common in patients with psoriasis than in those without it $[4,18-21]$.

The Swedish authors were the first to observe an increased incidence of obesity in the population of patients with psoriasis [21]. They conducted a retrospective study in a group of 159,200 people and found a relationship between obesity and psoriasis, especially in women.

Naldi et al. [19] observed a similar correlation in the group of 560 patients with psoriasis, where the incidence of psoriasis correlated with higher BMI and was two-fold more frequent in patients with elevated body weight. Sommer et al. [4] observed significantly higher BMI values in patients with psoriasis compared with controls, particularly in women. According to American authors [18], obesity and higher BMI values were more frequent in patients with psoriasis when compared with controls (34\% and 18\%, respectively). Herron et al. [18] concluded that increased body weight was not a risk factor for psoriasis but the consequence of the disease. This belief was confirmed by the results of Mallbris et al. [22], which showed no differences in BMI values in patients with recently diagnosed psoriasis (less than one year) and in the control group. However, as mentioned by HigaSansone et al. [23], a significant reduction in weight in a patient with morbid obesity resulted in complete clearance of psoriasis. According to Hamminga et al. [9], the first line treatment of psoriasis in obese patients should focus on weight loss to reduce severe inflammation associated with cytokines released by adipocytes. A strong association between excess body fat, obesity and psoriasis was also confirmed by Hercogova et al. [20]. In addition to the known relationship between the severity and incidence of psoriasis, the researchers emphasize the therapeutic implications for systemic therapy of psoriasis in patients with abnormal body weight. Murray et al. [24] studied the relationship between BMI and severity of psoriasis, expressed by body surface area (BSA) and psoriasis global assessment (PGA). In their study, psoriasis severity was found to be related to the degree of obesity. Psoriasis global assessment score increased with BMI and a positive correlation between BSA and BMI was observed. Patients with psoriasis were more likely to have a higher BMI, especially women.

In our study, patients with psoriasis had an increased prevalence of abnormal BMI compared with the control group. Seventy-two psoriatic patients (61.54\%) had an abnormal body mass index, 44 (36.06\%) were overweight and $28(22.9 \%)$ met the diagnostic criteria of central obesity by the International Diabetes Federation (IDF) [6]. In the control group, overweight was diagnosed in 14 patients (35\%) and obesity in 6 patients (15\%). However, in both groups, no correlation between BMI and severity of skin lesions (PASI) and nail changes (mNAPSI) was observed.

The consensus of the IDF has not only modified the definition of metabolic syndrome, but also highlighted a number of the other possible risk factors that appear to be related to this syndrome [2, 3]. According to this document, these additional factors could serve as a predictive factor for CVD and/or diabetes and should be included in the future research programs. The additional factors which should be considered include: cardiovascular disorders, adipose tissue biomarkers (leptin, adiponectin), prothrombotic state as well as proinflammatory state and elevated C-reactive protein levels [2].

In obesity, increased production of proinflammatory cytokines such as TNF- $\alpha$, interleukin IL-1, IL-6, IL-8 in adipose tissue has been observed [25]. The higher incidence of obesity in patients with psoriasis may result in severe inflammation, which can worsen the course of disease, increase the frequency of relapses and contribute to the development of severe metabolic disorders. C-reactive protein and ESR are well-known and widely available markers of inflammation. C-reactive protein is a sensitive marker of inflammation, synthesized and secreted by the liver. Its production is stimulated by interleukin-6, involved in the pathogenesis of many diseases, including psoriasis [8]. C-reactive protein seems to be a good marker of inflammation in psoriasis due to linkage of cytokines responsible for inflammation of the skin. An elevated level of CRP is always a result of interactions between inflammatory cytokines and their receptors and inhibitors [26].

Pardina et al. [27] studied the changes in levels of CRP, leptin, adiponectin, nitrogen oxide and insulin-like growth factor-1 (IGF-1) in patients with obesity and observed a highly significant decrease in elevated plasma CRP levels after bariatric surgery resulting in weight loss. Earlier studies indicate that in obese patients with no concomitant inflammatory diseases, chronic inflammation manifested by an elevated level of CRP and ESR can deteriorate the skin lesions in psoriasis [28, 29]. Chodorowska et al. [26] observed the changes in CRP levels in psoriatic patients during the disease exacerbation and after effective therapy of psoriasis. C-reactive protein levels were significantly higher during exacerbations than in the control group. In remission, CRP levels were reduced but still elevated compared to the control group and spontaneously reached normal values after a few weeks. Similarly, Gisondi et al. [30] observed higher values of CRP in patients with psoriasis than in controls. Moreover, higher levels of serum CRP were found in patients with psori- 
asis, related to the disease severity. The higher the PASI score, the higher the CRP level. The results of Coimbra et al. [31] remain in agreement with the above mentioned. They examined the activity of various inflammatory parameters, such as leukocytes, neutrophils, lactoferrin, elastase, $\alpha_{1}$-antitrypsin, and CRP. Compared with controls, they found higher levels of inflammatory markers in patients with psoriasis. In addition, a strong positive correlation between PASI and CRP was observed. According to the authors, CRP was a useful marker of psoriasis severity that could be used to monitor psoriasis and its treatment [31]. In contrast, Sergeant et al. [32] did not find CRP a marker of severity of psoriasis and a useful prognostic factor. In their study, elevated CRP levels were detected only in patients with psoriatic arthritis. There was no correlation between PASI and CRP levels. It was suggested that the result might be associated with a significant diversity of the study group, which included both patients with plaque psoriasis, pustular psoriasis and psoriatic arthritis.

Interestingly, in our study the correlation between CRP levels and PASI and mNAPSI scores was not observed, although our group of patients with psoriasis was relatively homogeneous. Only 11 patients (9.4\%) had psoriasis arthritis, which does not appear to have an impact on the results. Patients with psoriasis had significantly higher CRP levels than healthy controls. Other possible causes of elevated levels of this protein were excluded, particularly inflammatory liver diseases.

According to many authors, CRP is actively involved in the inflammation process, inhibits neutrophil chemotaxis and adhesion to the endothelium and reduces migration to inflammatory foci and not merely manifests the inflammatory state. Moreover, it is considered a risk factor for atherosclerosis [15, 31] and cardiovascular diseases [33]. Another marker of inflammation of a high clinical significance is the ESR. Rocha-Pereira et al. [34] studied a number of inflammation factors, including the erythrocyte sedimentation rate, in patients with psoriasis. Compared to the control group, ESR was elevated in psoriatic patients, both during exacerbation and remission of the disease. The ESR level was higher in the active phase of psoriasis.

In our study, patients with psoriasis had significantly higher ESR levels than those in the control group. Moreover, there was a positive correlation between ESR and $\mathrm{BMI}$ both in patients with psoriasis and in controls. As Froom et al. [35] suggested, persistently elevated ESR might be a risk factor for the development of many diseases such as psoriasis, myocardial infarction, ankylosing spondylitis, inflammatory bowel disease; but in our study, no correlation between the severity of psoriasis (PASI and mNAPSI) and ESR values was observed.

It is believed that the risk of metabolic disorders and associated cardiovascular diseases in psoriatic patients can be reduced through appropriate treatment of psoriasis [36], because prompt and effective psoriasis therapy can reduce inflammation, an important component of the metabolic syndrome.

Hypertension is one of the important criteria for the diagnosis of metabolic syndrome. Cohen et al. [37] showed higher blood pressure in patients with psoriasis compared with the control group and suggested that psoriatic patients should be routinely screened for hypertension. The results of Neimann et al. [38] demonstrated raised blood pressure, higher incidence of diabetes and lipid abnormalities in patients with psoriasis. Patients with severe psoriasis were more vulnerable to the incidence of risk factors for cardiovascular disease, especially hypertension, obesity or type 2 diabetes. Sommer et al. [4] confirmed that the occurrence of some chronic disorders could be associated with psoriasis. In psoriatic patients, type 2 diabetes, dyslipidemia and coronary heart disease were more common compared with healthy subjects. However, according to Gisondi et al. [39], blood pressure values, glycemia and plasma high-density lipoprotein (HDL) cholesterol levels were similar in psoriatic patients and controls. In our study, a significantly higher incidence of hypertension in patients with psoriasis compared with the control group was found. Psoriatic patients had significantly higher both systolic and diastolic blood pressure.

An elevated level of blood glucose is an important criterion for the diagnosis of metabolic syndrome. Nisa and Qazi [6] and Grzybowski et al. [40] observed significantly higher blood glucose levels in patients with psoriasis than in healthy subjects, whereas Gisondi et al. [39] and Janusz et al. [41] obtained the opposite results. Janusz et al. [41] tried to assess the prevalence of insulin resistance in patients with psoriasis and found that despite high levels of insulin in the blood, the glucose concentration was comparable to that in the control group. Peserico et al. [42] put forward an interesting hypothesis. In their study, elevated levels of blood glucose, hypertension and lipid disorders were observed only in obese psoriatic patients. Such findings suggest that only overweight psoriatic patients exhibit the metabolic abnormalities frequently reported in the literature, while psoriatic patients of normal weight do not differ from the general population. In our study there was no difference in prevalence of abnormal glucose levels in patients with psoriasis compared to the control group.

Psoriasis is a disease of a complex pathogenesis and may coexist with other diseases, apparently not related to one another. Several recent studies found an increased incidence of non-alcoholic fatty liver disease (NAFLD) in psoriasis patients [43]. In the light of recent findings, NAFLD is now regarded as the hepatic manifestation of metabolic syndrome and represents the most common cause of abnormal serum liver enzymes [44]. It is well known that in patients with psoriasis, the incidence of obesity and metabolic syndrome is more common than in healthy population $[4,38,39,43]$ and these are two 
well-known risk factors for NAFLD. The potential underlying mechanism may include the presence of chronic inflammation what promotes the development of insulin resistance [45], which is considered the most important pathogenetic factor for NAFLD.

Gisondi et al. [44] stated that inflammatory liver disease, including NAFLD might actively contribute to the severity of psoriasis through the release of pathogenetic mediators from the inflamed liver. In their study, the presence of NAFLD was the only significant predictor of higher PASI score, irrespective of age, sex, BMI, duration of psoriasis. In our study, patients with psoriasis and concomitant hepatic dysfunction were observed. Patients with increased liver enzyme levels had the De Ritis ratio calculated, which is considered a useful marker to differentiate nonalcoholic steatohepatitis (NASH), NAFLD, alcoholic liver disease and others [46].

Patients suffering from psoriasis and liver dysfunction manifested some differences compared with psoriatic patients without it. Patients with a calculated De Ritis ratio had significantly higher body weight and BMI than those with normal levels of liver enzymes. Interestingly, there was no significant increase in inflammatory markers - CRP and ESR - in patients with a suspected liver dysfunction, which is likely to be associated with an early stage of liver disease, the only abnormality were elevated liver enzymes. However, the increased prevalence of obesity and higher BMI values in these patients may be risk factors for development and progression of the disease.

The literature data clearly indicated a relationship between psoriasis and components of the metabolic syndrome. Many studies also confirmed a higher incidence of metabolic syndrome in patients with psoriasis [4, 39]. It seems that psoriasis is primarily associated with a higher prevalence of some risk factors, irrespective of the full manifestation of the metabolic syndrome. The results obtained in our study indicate that psoriatic patients appear to be at a higher risk of the metabolic disorders such as hypertension, obesity, liver dysfunction, increased inflammatory parameters than healthy subjects. We suggest that patients with psoriasis should be routinely screened for the presence of the above-mentioned disorders.

\section{References}

1. Szczeklik A. Internal medicine [Polish]. Practical Medicine, Krakow 2005.

2. Alberti KG, Zimmet P, Shaw J. Metabolic syndrome - a new world-wide definition. A Consensus Statement from the International Diabetes Federation. Diabet Med 2006; 23: 469-80.

3. Korzon-Burakowska A, Dziemidok P. Diabetic foot - the need for comprehensive multidisciplinary approach. Ann Agric Environ Med 2011; 18: 314-7.

4. Sommer DM, Jenisch S, Suchan M, et al. Increased prevalence of the metabolic syndrome in patients with moderate to severe psoriasis. Arch Dermatol Res 2006; 298: 321-8.
5. Christophers E. Comorbidities in psoriasis. Chris Clin Dermatol 2007; 25: 529-34.

6. Nisa N, Qazi MA. Prevalence of metabolic syndrome in patients with psoriasis. Indian J Dermatol Venereol Leprol 2010; 76: 662-5.

7. Schoelson SE, Lee J, Goldfine AB. Inflammation and insulin resistance. J Clin Invest 2006; 116: 1793-801.

8. Wolska H, Langner A. Psoriasis [Polish]. Wydawnictwo Czelej, Lublin 2006.

9 Hamminga EA, van der Lely AJ, Neumann HA, Thio HB. Chronic inflammation in psoriasis and obesity: implications for therapy. Med Hypotheses 2006; 67: 768-73.

10. Henseler T, Christophers E. Disease concomitance in psoriasis. J Am Acad Dermatol 1995; 32: 982-6.

11. Alexander E, Pinto J, Pal GS, et al. Disease concomitance in psoriasis. A clinical study of 61 cases. Indian J Dermatol Venerol Leprol 2001; 67: 66-8.

12. Gulliver W. Long-term prognosis in patients with psoriasis. Br J Derm 2008; 159 Suppl 2: 2-9.

13. Griffiths CEM, Christophers E, Barker JNWN, et al. A classifi cation of psoriasis vulgaris according to phenotype. Br J Dermatol 2007; 156: 258-62.

14. Pietrzak A, Chodorowska G, Szepietowski J, et al. Psoriasis and serum lipid abnormalities. Dermatol Ther 2010; 23: 160-73.

15. Scirica BM, Morrow DA. Is C-reactive protein an innocent bystander or proatherogenic culprit? The verdict is still out. Circulation 2006; 113: 2128-34.

16. Haukeland JW, Damls JK, Konopski Z, et al. Systemic inflammation in nonalcoholic fatty liver disease is characterized by elevated levels of CCL2. J Hepatol 2006; 44: 1167-74.

17. Wyrzykowski B, Zdrojewski T, Syganowska E, et al. Epidemiology of metabolic syndrome in Poland. Results of the WOBASZ program. Kardiol Pol 2005; 63 (6 Suppl 4): S1-4.

18. Herron MD, Hinckley M, Hoffman MS, et al. Impact of obesity and smoking on psoriasis presentation and management. Arch Dermatol 2005; 141: 1527-34.

19. Naldi L, Chatenoud L, Linder D, et al. Cigarette smoking, body mass index, and stressful life events as risk factors for psoriasis: results from an Italian case-control study. J Invest Dermatol 2005; 125: 61-7.

20. Hercogová J, Ricceri F, Tripo L, et al. Psoriasis and body mass index. Dermatol Ther 2010; 23: 152-4.

21. Lindegard B. Diseases associated with psoriasis in a general population of 159,200 middle-aged, urban, native Swedes. Dermatologica 1986; 172: 298-304.

22. Mallbris L, Granath F, Hamsten A, Stahle M. Psoriasis is associated with lipid abnormalities at the onset of skin disease. J Am Acad Dermatol 2006; 54: 614-21.

23. Higa-Sansone G, Szomstein S, Soto F, et al. Psoriasis remission after laparoscopic Roux-en-Y gastric bypass for morbid obesity. Obes Surg 2004; 14: 1132-4.

24. Murray ML, Bergstresser PR, Adams-Huet B, Cohen JB. Relationship of psoriasis severity to obesity using same-gender siblings as controls for obesity. Clin Exp Dermatol 2009; 34: $140-4$

25. Arican O, Aral M, Sasmaz S, Ciragil P. Serum levels of TNFalpha, IFN-gamma, IL-6, IL-8, IL-12, IL-17, and IL-18 in patients with active psoriasis and correlation with disease severity. Mediators Inflamm 2005; 2005: 273-9.

26. Chodorowska G, Wojnowska D, Juszkiewicz-Borowiec $M$. C-reactive protein and alpha2-macroglobulin plasma activity in medium-severe and severe psoriasis. J Eur Acad Dermatol Venereol 2004; 18: 180-3.

27. Pardina E, Ferrer R, Baena-Fustegueras JA, et al. The relationships between IGF-1 and CRP, NO, leptin, and adiponec- 
tin during weight loss in the morbidly obese. Obes Surg 2010; 20: 623-32.

28. Visser M, Bouter LM, McQuillan GM, et al. Elevated C-reactive protein levels in overweight and obese adults. JAMA 1999; 282: 2131-5.

29. Pasulka PS, Bistrian BR, Blackburn GL. Obesity and erythrocyte sedimentation rates. Ann Intern Med 1985; 103: 304.

30. Gisondi P, Malerba M, Malara G, et al. C-reactive protein and markers for thrombophilia in patients with chronic plaque psoriasis. Int I Immunopathol Pharmacol 2010; 23: 1195-202.

31. Coimbra S, Oliveira H, Reis F, et al. C-reactive protein and leucocyte activation in psoriasis vulgaris according to severity and therapy. J Eur Acad Dermatol Venereol 2010; 24: 789-96.

32. Sergeant A, Makrygeorgou A, Chan WC, et al. C-reactive protein in psoriasis. Br J Dermatol 2008; 158: 417-9.

33. Ridker PM. High-sensitivity C-reactive protein: potential adjunct for global risk assessment in the primary prevention of cardiovascular disease. Circulation 2001; 103: 1813-8.

34. Rocha-Pereira P, Santos-Silva A, Rebelo I, et al. The inflammatory response in mild and in severe psoriasis. Br J Dermatol 2004; 150: 917-28.

35. Froom P, Margaliot S, Caine Y, Benbassat J. Significance of erythrocyte sedimentation rate in young adults. Am J Clin Pathol 1984; 82: 198-200.

36. Prodanovich S, Ma F, Taylor JR, et al. Methotrexate reduces incidence of vascular diseases in veterans with psoriasis or rheumatoid arthritis. J Am Acad Dermatol 2005; 52: 262-7.

37. Cohen AD, Weitzman D, Dreiher J. Psoriasis and hypertension: a case-control study. Acta Derm Venereol 2010; 90: 23-6.

38. Neimann AL, Shin DB, Wang X, et al. Prevalence of cardiovascular risk factors in patients with psoriasis. J Am Acad Dermatol 2006; 55: 829-35.

39. Gisondi P, Tessari G, Conti A, et al. Prevalence of metabolic syndrome in patients with psoriasis: a hospital-based casecontrol study. Br J Dermatol 2007; 157: 68-73.

40. Grzybowski G, Fafara I, Żaba R, Wierusz-Wysocka B. Evaluation of glucose, insulin, C-peptide uric acid serum levels in patients with psoriasis. Pol Merkur Lekarski 2001; 11: 495-8.

41. Janusz I, Lewandowski K, Lukamowicz J, et al. Insulin resistance and adiponectin levels in psoriasis patients. Post Dermatol Alergol 2010; 27: 451-5.

42. Peserico A, Zanetti G, Padovan S, et al. Relationship between body weight and blood pressure and some metabolic parameters in psoriatic patients. Br J Dermatol 1988; 118: 191-4.

43. Wenk K, Arrington K, Ehrlich A. Psoriasis and non-alcoholic fatty liver disease. J Eur Acad Dermatol Venereol 2011; 25: 383-91.

44. Gisondi P, Targher G, Zoppini G, Girolomoni G. Non-alcoholic fatty liver disease in patients with chronic plaque psoriasis. J Hepatol 2009; 51: 758-64.

45. Shoelson SE, Herrero L, Naaz A. Obesity, inflammation, and insulin resistance. Gastroenterology 2007; 132: 2169-80.

46. Sorbi D, Boynton J, Lindor KD. The ratio of aspartate aminotransferase to alanine aminotransferase: potential value in differentiating nonalcoholic steatohepatitis from alcoholic liver disease. Am J Gastroenterol 1999; 94: 1018. 